\title{
Assess Before Rx: Reducing the Overtreatment of Asymptomatic Blood Pressure Elevation in the Inpatient Setting
}

\author{
Sara D Pasik, BA'; Sophia Chiu, MS'; Jeong Yang, BA'; Catherine Sinfield, $\mathrm{MPH}^{1}$; Nicole Zubizarreta, $\mathrm{MPH}^{2}$; \\ Rosemarie Ramkeesoon, FNP3; Hyung J Cho, MD4; Mona Krouss, MD*
}

'Department of Medical Education, Icahn School of Medicine at Mount Sinai, New York, New York; ${ }^{2}$ Department of Population Health Science and Policy, Institute for Healthcare Delivery Science, Icahn School of Medicine at Mount Sinai, New York, New York; ${ }^{3}$ Department of Nursing, Mount Sinai Hospital, New York, New York; ' ${ }^{4}$ epartment of Medicine, Icahn School of Medicine at Mount Sinai, New York, New York.

BACKGROUND: Asymptomatic blood pressure elevation is common in the inpatient setting. National guidelines recommend treating with oral agents to slowly decrease blood pressure; however, many clinicians use intravenous antihypertensive medications, which can lead to unpredictable changes in blood pressure.

OBJECTIVE: To decrease the number of inappropriate orders (without symptoms of hypertensive emergency or order for NPO) of intravenous antihypertensives and adverse events associated with intravenous orders.

DESIGN: Quasi-experimental study with multidisciplinary intervention.

PARTICIPANTS: Inpatients with a one-time order for an intravenous antihypertensive agent from January 2016 to February 2018.
MAIN MEASURES: The main outcomes were the total numbers of orders and inappropriate orders, adverse events, and alternate etiologies per 1,000 patient-days. As a balancing measure, patients were monitored for adverse events when blood pressure was elevated and not treated.

KEY RESULTS: There were a total of 260 one-time orders of intravenous antihypertensives on two medical units. Inappropriate orders decreased from 8.3 to 3.3 per 1,000 patient days $(P=.0099)$. Adverse events associated with intravenous antihypertensives decreased from 3.7 to 0.8 per 1,000 patient days $(P=.0072)$.

CONCLUSION: This initiative demonstrated a significant reduction in inappropriate use of IV antihypertensives and an associated reduction in adverse events. Journal of Hospital Medicine 2019;14:151-156. (c) 2019 Society of Hospital Medicine
W ith the presence of hypertension in $25 \%$ of patients admitted to the hospital, ${ }^{1}$ its proper management is imperative. A hypertensive crisis is a severe elevation of blood pressure, defined as systolic $\geq 180$ $\mathrm{mm} \mathrm{Hg}$ and/or diastolic $\geq 120 \mathrm{~mm} \mathrm{Hg}$. It is further classified as either a hypertensive emergency which includes the presence of end-organ damage, ${ }^{2}$ or hypertensive urgency, defined as asymptomatic blood pressure elevation. ${ }^{3}$ Although hypertensive emergencies account for only $1 \%-2 \%$ of patients with hypertension, ${ }^{4}$ they are associated with a high one-year mortality rate $(>79 \%){ }^{5}$ Hypertensive emergency requires immediate reduction of blood pressure with IV antihypertensive drugs to limit organ damage. In contrast, as per national guidelines, inpatient management of hypertensive urgency requires gradual reductions of blood pressure over hours to days using oral antihypertensives. ${ }^{2}$ It is also recommended that alternative etiologies, such as anxiety or pain, be considered before treatment is initiated. ${ }^{1}$

*Corresponding Author: Mona Krouss, MD, E-mail: mona.krouss@mountsinai. org; Telephone: 212-241-3097; Twitter: @kroussMD.

Received: September 2, 2018; Revised: October 30, 2018;

Accepted: November 8, 2018

๑ 2019 Society of Hospital Medicine DOI 10.12788/jhm.3190
Clinicians often inappropriately treat asymptomatic hypertension in the inpatient setting, ${ }^{6,7}$ using intravenous (IV) antihypertensive medications despite evidence showing potential harm. ${ }^{5,8}$ This can lead to unpredictable reductions in blood pressure. ${ }^{79} \mathrm{~A}$ recent retrospective analysis demonstrated that $32.6 \%$ of patients had a blood pressure reduction greater than $25 \%$ after the use of an IV antihypertensive. ${ }^{7}$ Reductions greater than $25 \%$ lead to shifts in autoregulation, which may result in patient harm, such as hypotension, decreased renal perfusion, and stroke. IV medications are also more expensive than oral agents, due to the additional cost of administration.

Although overtreatment of asymptomatic hypertension with IV antihypertensive medications is common, initiatives to address this in inpatient settings are lacking in the literature. The aim of this quality improvement initiative was to reduce unnecessary IV antihypertensive treatment for hypertensive urgency in the inpatient setting.

\section{METHODS}

\section{Setting}

An interdisciplinary quality improvement intervention was initiated on two inpatient medicine units at an urban, 1,134-bed tertiary medical center affiliated with the Icahn School of Medicine at Mount Sinai. Members of the Mount Sinai High Value 
Care Committee and the Student High Value Care Initiative ${ }^{10}$ developed this project. The intervention was implemented in stages from March 2017 to February 2018. It targeted nurses, housestaff, nurse practitioners, and attendings on general medical teaching and nonteaching services. The components of the intervention included education, a treatment algorithm, audit and feedback, and electronic medical record (EMR) change. This project was submitted to the Quality Committee in the Department of Medicine and determined to be a quality improvement project rather than research and thus, an IRB submission was not required.

\section{Treatment Algorithm and Education}

A clinical algorithm was designed with nursing and cardiology representatives to provide guidance for nurses regarding the best practice for evaluation of inpatient hypertension, focusing on assessing patients before recommending treatment ("Assess Before Rx"; Figure 1). Educational sessions reinforcing the clinical algorithm were held monthly at nursing huddles. These involved an introduction session providing the background and purpose of the project, with follow-up sessions including interactive mock cases on the assessment of hypertensive urgency.

A second treatment algorithm was designed, with housestaff and cardiology input, to provide guidance for the internal medicine housestaff and nurse practitioners. It utilized a similar approach regarding identification, evaluation, and assessment of alternate etiologies but included more detailed treatment recommendations with a table outlining the oral medications used for hypertensive urgency (Figure 2). The flowchart and table were uploaded to an existing mobile application used by housestaff and nurse practitioners for quick access. The mobile application is frequently used by housestaff and contains many clinical resources. Additionally, e-mails including the purpose of the project and the treatment algorithm were sent to rotating housestaff at the start of each new medicine rotation.

\section{Audit and Feedback}

Monthly feedback was e-mailed to the nurses, which reinforced the goals and provided positive feedback on outcomes with an announcement of the "Nurse of the Month." The winners were selected based on the most accurate and appropriate documentation of their assessments determined through retrospective chart review.

Targeted e-mail feedback was also sent to providers who ordered IV antihypertensives without the appropriate indication. The e-mails included the medical record number, date and time of the order, any alternate etiologies that were documented, and any adverse events that occurred as a result of the medication.

\section{Systems Change: Electronic Medical Record Orders} EMR advisory warnings were placed on IV antihypertensive orders of labetalol and hydralazine. The alerts served to nonintrusively remind providers to assess for symptoms before placing the order to ensure that the order was appropriate.

\section{Data Collection and Assessment}

Seven-month preintervention (January-July 2016) and 12-month postintervention (March 2017-February 2018) data were compared. The months prior to intervention were excluded to account for project development and educational lag. Data were obtained from EMR utilization reports of onetime orders of IV labetalol and hydralazine, and retrospective chart review. Patients who were pregnant, less than 18 years of age, or postoperative were excluded. Orders were designated as inappropriate if there was no evidence of hypertensive emergency through documentation in progress notes, or if the patient was able to take oral medication (not NPO). Adverse events were defined as a blood pressure drop of more than $25 \%$, a change in the heart rate by more than 20 beats per minute, or the need for IV fluids, based on previous studies. ${ }^{7}$ Although decreased blood pressure is not necessarily dangerous in and of itself, adverse events arising from blood pressure decreasing too rapidly from IV antihypertensives are well documented. ${ }^{9,11}$ The presence of alternate etiologies of high blood pressure that were documented in progress notes, including pain, anxiety, agitation, and holding of home blood pressure medications, were recorded. The numbers of inappropriate orders pre- and postintervention were compared. Confounding factors of patient age and length of stay (LOS) were compared pre- and postintervention in order to rule out other factors to which the intervention's effect could be attributed. Additionally, as a balancing measure, a random sample of patients with elevated blood pressure were monitored on a biweekly basis for adverse events that occurred as a result of not receiving IV treatment, including stroke, myocardial infarction, and pulmonary edema.

For this study, orders were reported on the standardized form of orders per 1,000 patient days. This was calculated as the number of orders divided by the total number of patient days from the two medicine units. For the univariate analysis, pre- and postintervention orders were compared for the different order categories using a t-test. Results were considered statistically significant at $P<.05$. Data analysis was conducted using SAS v. 9.4 (SAS Institute, Cary, North Carolina).

Additionally, a cost analysis was performed to estimate the hospital-wide annual cost of inappropriate orders. The analysis used the cost per dose ${ }^{12}$ and included nurse-time derived from the median salary of those on our units. The hospital-wide cost was extrapolated to estimate the potential annual savings for the institution.

\section{RESULTS}

A total of 260 one-time orders of IV antihypertensives were analyzed in this study, 127 in the seven-month preintervention period and 133 in the 12-month postintervention period. The majority, $67.3 \%$ ( $n=175)$, were labetalol orders. Inappropriate orders (ie, neither NPO nor hypertensive emergency) decreased from 8.3 to 3.3 orders per 1,000 patient days $(P=$ .0099; Figure 3).

In total, there were 86 adverse events (33.1\%), the majority of which $(94.2 \%, n=81)$ were a $>25 \%$ decrease in blood pressure 


\section{Assess before Rx}

Did you know...

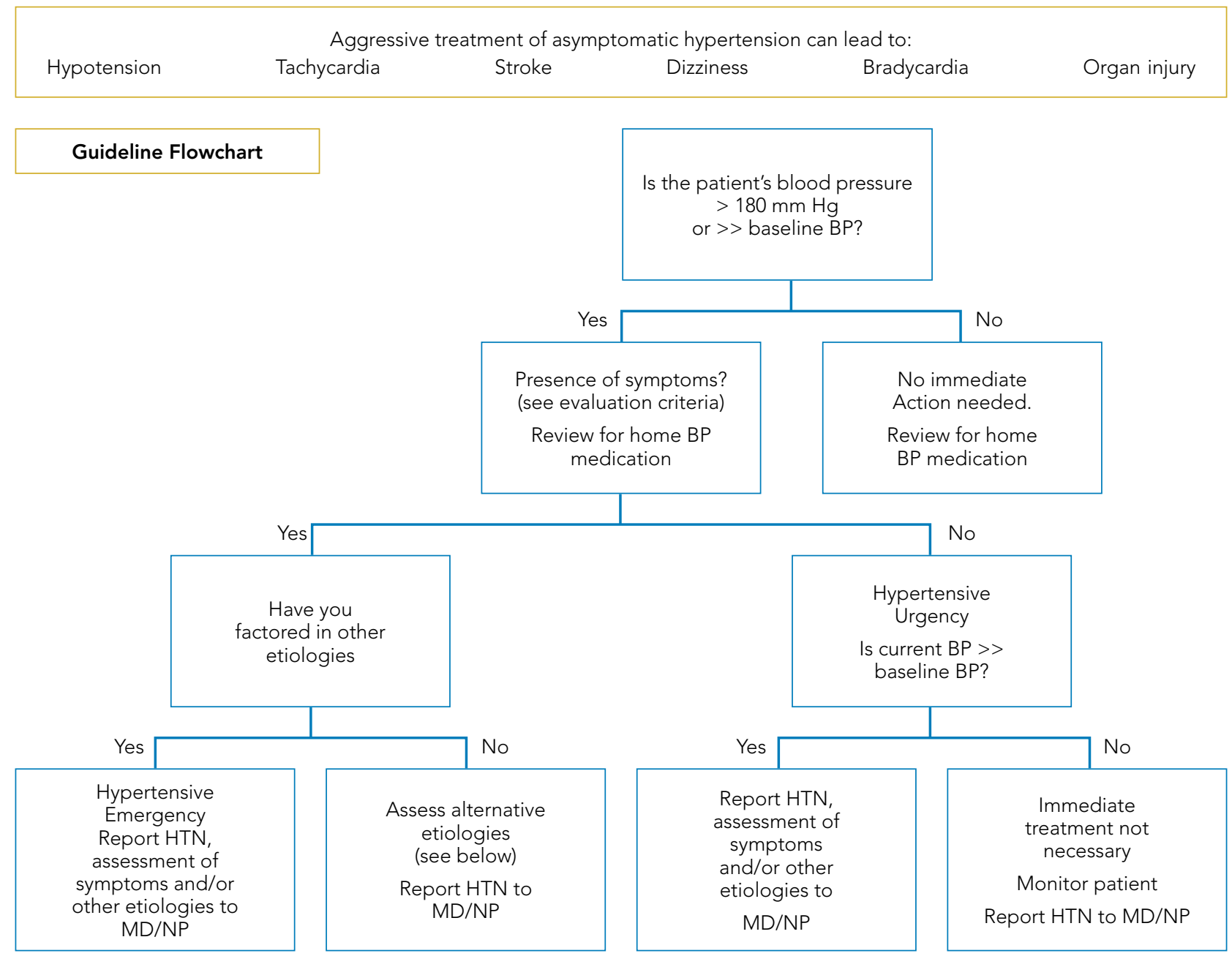

\section{Evaluation Criteria}

1. Check vitals (BP in both arms)

2. Check for the following:

- Neuro check

- Symptoms

- Altered mental status

- Headache

- Changes in vision

- Chest pain

- Shortness of breath

- Acute stroke

3. Check if anti-HTN meds were recently held

\section{Alternative Etiologies}

- Missing home BP meds

- Drug withdrawal

- Anxiety/Pain

- Delirium

- Volume overload (especially with renal/cardiac patients)

FIG 1. Nurse Inpatient Hypertension Guideline

Abbreviations: BP, blood pressure; HTN, hypertension; MD/NP, doctor of medicine/nurse practitioner.

(Table 1). The number of adverse events per 1,000 patient days decreased from 4.4 in the preintervention period to 1.9 postin- tervention, $P=.0112$. Of the inappropriate orders, adverse events decreased from 3.7 to 0.8 per 1,000 patient days, $P=$ 


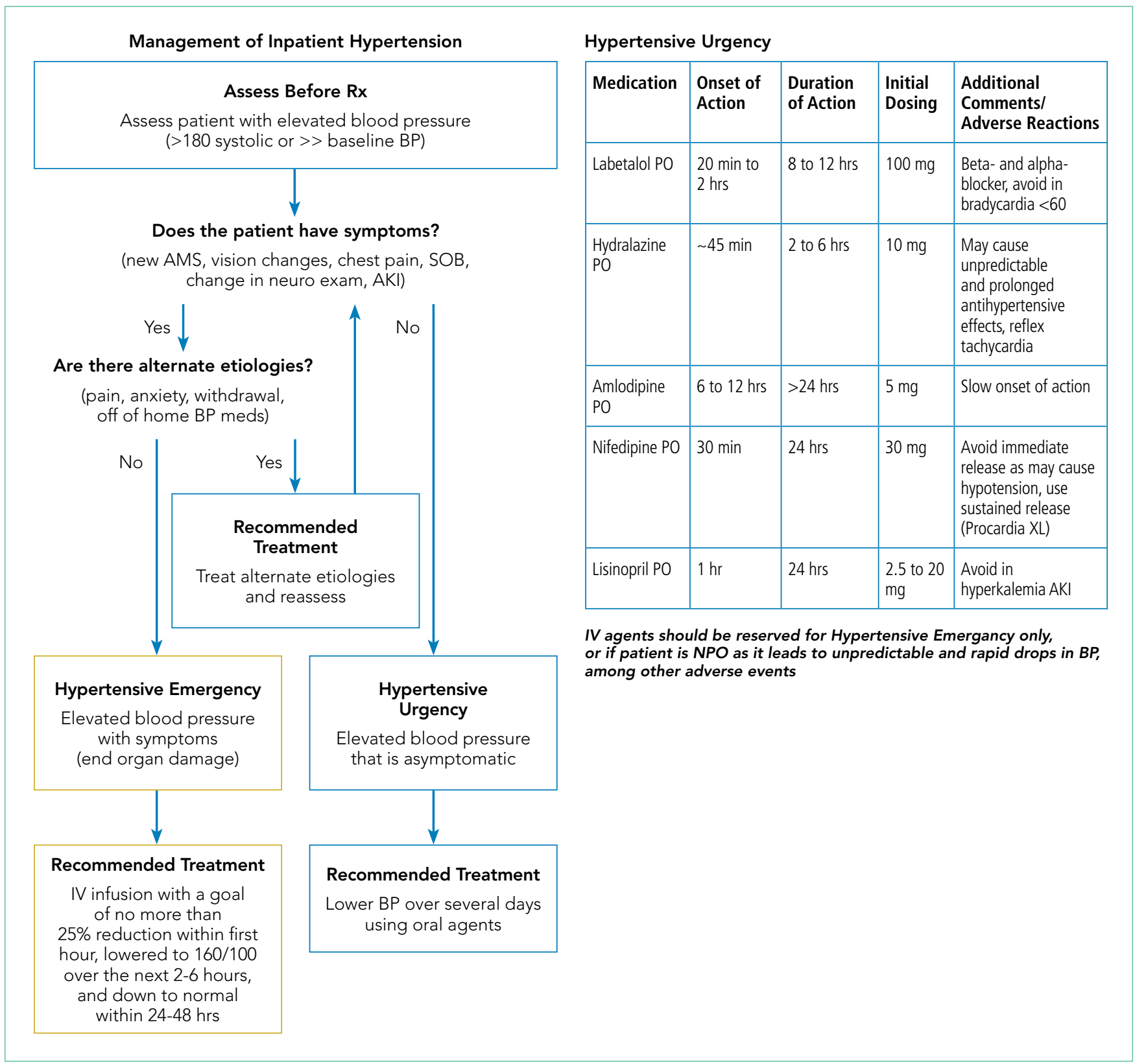

FIG 2. Housestaff Inpatient Hypertension Management Guidelines

Abbreviations: AKI, acute kidney injury; AMS, acute mountain syndrome; BP, blood pressure; IV, intravenous; NPO, nothing by mouth; PO, by mouth; SOB, shortness of breath.

.0072. Overall, there were 76 orders (29.2\%) with documented alternate etiologies. The number of orders per 1,000 patient days with an alternate etiology decreased from 4.7 in the preintervention period to 1.2 postintervention, $P=.0044$ (Table 2). Descriptive analysis of patient characteristics pre- and postintervention were not statistically significant; for age 68.4 vs 70.7, $P=.0823$ and for LOS 14.8 vs $15.4, P=.0769$. As a balancing measure, 111 patients with elevated blood pressure were monitored for adverse events during the postintervention period. Among patients who did not receive IV medication based on our algorithm, there were no adverse events.

Cost analysis estimated a $\$ 17,890$ annual hospital-wide cost for unnecessary IV antihypertensive medications before the intervention. The estimate was calculated using the number of orders on the two medical units observed during the seven-month preintervention period, extrapolated to a 12-month period and to the total number of 15 medical units in the hospital. The intervention on the two studied medical units themselves led to an estimated $\$ 1,421$ cost reduction (59.6\%). Had the intervention been implemented hospital-wide with similar results, the resulting cost reduction would have amounted to $\$ 10,662$.

\section{DISCUSSION}

Our initiative successfully demonstrated a significant reduction of $60 \%$ in inappropriate one-time orders of IV antihyperten- 


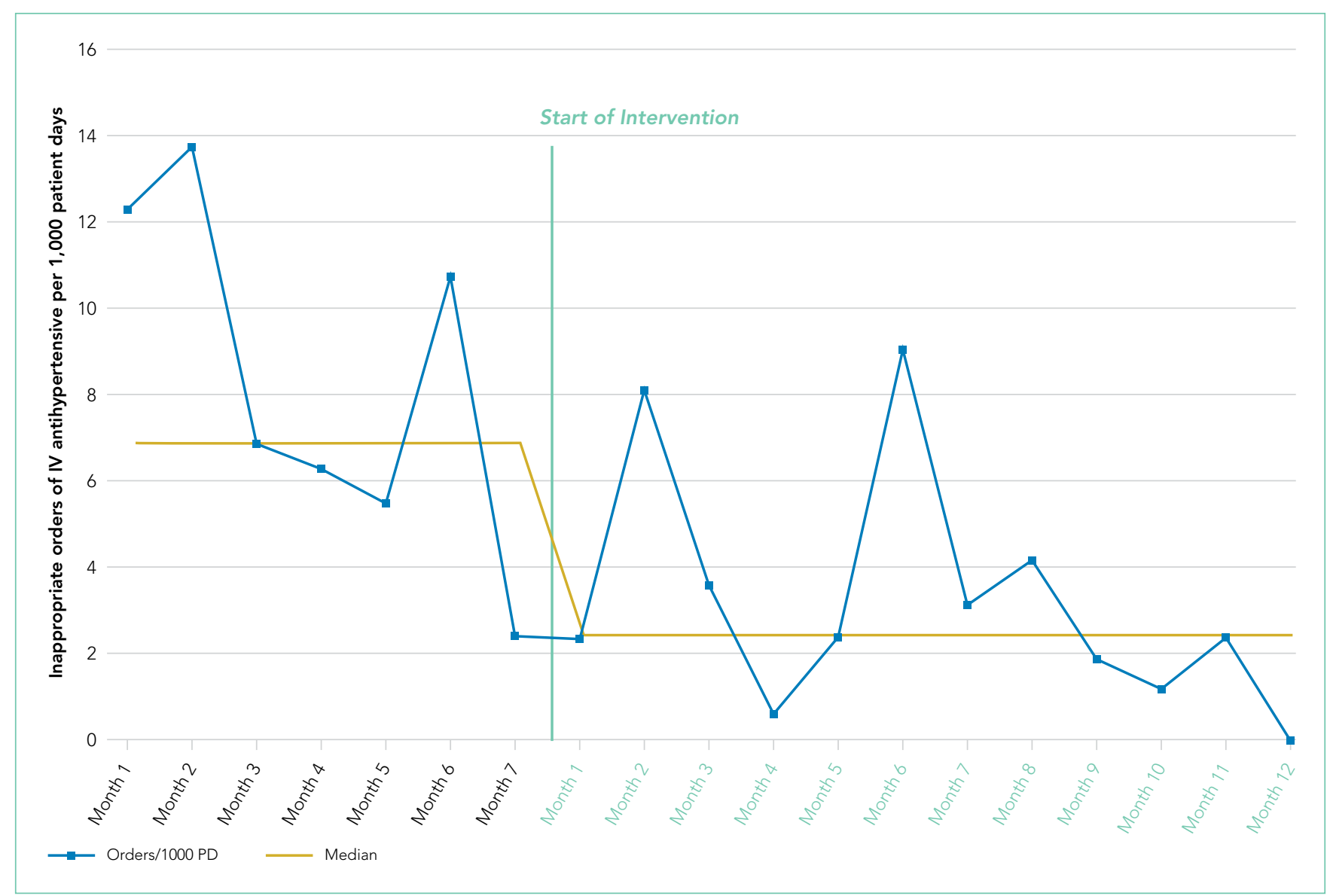

FIG 3. U-Control Chart, Pre- and Postintervention Inappropriate Orders per 1,000 Patient Days

sives per 1,000 patient days. Accordingly, the number of adverse events per 1,000 patient days decreased by $57 \%$. There was also a decrease in the number and percentage of IV orders with documented alternate etiologies. We hypothesize that this was due to nurses and physicians assessing and treating these conditions prior to treating hypertension in the intervention period, consequently avoiding an IV order.

The goal of the intervention was to have nurses assess for end-organ damage and alternate etiologies and include this information on their assessment provided to the physician, which would result in appropriate treatment of elevated blood pressure. By performing an interdisciplinary intervention, we addressed the knowledge deficit of both nurses and physicians, improved the triage of elevated blood pressure, and likely decreased the number of pages to providers.

To our knowledge, this is the first intervention addressing the inpatient overuse of IV antihypertensive medications for the treatment of asymptomatic hypertension. Additionally, this study bolsters prior evidence that the use of IV antihypertensives in asymptomatic patients leads to a large number of adverse events. ${ }^{7}$ A third of patients in the preintervention period had documented alternate etiologies of their blood pressure elevation, highlighting the need to assess and potentially treat these causes prior to treating blood pressure itself.

Reducing unnecessary treatment of asymptomatic blood
TABLE 1. Adverse Events and Alternative Etiology of IV Antihypertensive Medications

\begin{tabular}{lc}
\hline Adverse Events & $\mathbf{n}(\%)$ \\
\hline Blood Pressure Drop $>25 \%$ & $81(94.2)$ \\
\hline Bradycardia & $6(7.0)$ \\
\hline Tachycardia & $2(2.3)$ \\
\hline Symptomatic Dizziness & $2(2.3)$ \\
\hline Need for IV Fluids & $0(0.0)$ \\
\hline Alternative Etiologya & $\mathbf{n}(\%)$ \\
\hline Anxiety & $22(28.9)$ \\
\hline Pain & $38(50.0)$ \\
\hline Steroids & $5(6.6)$ \\
\hline Withdrawal & $1(1.3)$ \\
\hline Off Home Antihypertensives & $10(13.2)$ \\
\hline aNot mutually exclusive & \\
Abbreviation: IV, intravenous. &
\end{tabular}


TABLE 2. Analyses of One-Time Orders of IV Antihypertensive Medications

\begin{tabular}{|c|c|c|c|}
\hline & Pre Intervention (7 months) & Post Intervention (12 months) & $P$ Value \\
\hline \multicolumn{4}{|l|}{ Adverse Events / 1,000 patient days } \\
\hline From All Orders & 4.4 & 1.9 & .0112 \\
\hline Alternate Etiology Orders / 1,000 patient days & 4.7 & 1.2 & .0044 \\
\hline
\end{tabular}

pressure elevation is challenging. Evidence shows that both clinicians and patients overestimate the benefits and underestimate the harms of medical interventions. ${ }^{13,14}$ This unfortunately leads to unjustified enthusiasm for medical treatments, which can worsen outcomes. ${ }^{15}$ Additionally, there may be a lack of knowledge of the guidelines, as well as the amount of time required in the full assessment of hypertensive urgency, that creates a culture of "treating the number."

Changing physician behavior is difficult. ${ }^{16}$ However, active forms of continuing education and multifaceted interventions, such as ours, are most effective. ${ }^{17}$ Our message focused on patient safety and harm reduction, addressed clinicians' safety concerns, and included stories of real cases where this overuse led to adverse events-all of which are encouraged in order to facilitate clinician engagement. ${ }^{18}$

There were limitations to this study. Only blood pressure elevations associated with an IV antihypertensive order and not all blood pressure elevations meeting the criteria for hy-

\section{References}

1. Herzog $E$, Frankenberger $O$, Aziz $E$, et al. A novel pathway for the management of hypertension for hospitalized patients. Crit Pathw Cardiol. 2007;6(4):150-160. doi: 10.1097/HPC.0b013e318160c3a7.

2. Whelton PK, Carey RM, Aronow WS, et al. 2017 ACC/AHA/AAPA/ABC/ ACPM/AGS/APhA/ASH/ASPC/NMA/PCNA guideline for the prevention, detection, evaluation, and management of high blood pressure in adults: a report of the American College of Cardiology/American Heart Association Task Force on clinical practice guidelines. Hypertension. 2018;71(6):e13-e115. doi: 10.1161/HYP.0000000000000065.

3. Mancia G, Fagard R, Narkiewicz K, et al. 2013 ESH/ESC guidelines for the management of arterial hypertension: the Task Force for the Management of Arterial Hypertension of the European Society of Hypertension (ESH) and of the European Society of Cardiology (ESC). Eur Heart J. 2013;34(28):21592219. doi: 10.1093/eurheartj/eht151.

4. Global status report on noncommunicable diseases 2010. Geneva, Switzerland: World Health Organization; 2011. 3.

5. Weder $A B$, Erickson $S$. Treatment of hypertension in the inpatient setting: use of intravenous labetalol and hydralazine. J Clin Hypertens (Greenwich). 2010;12(1):29-33. doi: 10.1111/j.1751-7176.2009.00196.x.

6. James PA, Oparil S, Carter BL, et al. 2014 evidence-based guideline for the management of high blood pressure in adults: report from the panel members appointed to the Eighth Joint National Committee (JNC 8). JAMA. 2014;311(5):507-520. doi: 10.1001/jama.2013.284427.

7. Lipari M, Moser LR, Petrovitch EA, Farber M, Flack JM. As-needed intravenous antihypertensive therapy and blood pressure control. J Hosp Med. 2016;11(3):193-198. doi: 10.1002/jhm.2510.

8. Patel KK, Young L, Howell EH, et al. Characteristics and outcomes of patients presenting with hypertensive urgency in the office setting. JAMA Intern Med. pertensive urgency in general were examined. Additionally, our documentation of symptoms of hypertensive emergency and alternate etiologies was based only on documentation in the medical record. Ideally, we would have liked to conduct an interrupted time series analysis to assess the effect of the intervention over time; however, there were not enough orders of IV antihypertensives to perform such an analysis.

\section{CONCLUSION}

Treatment of asymptomatic blood pressure with IV antihypertensive medications can lead to patient harm. To reduce inappropriate treatment, our Student High Value Care team set out to challenge this common practice. Our interdisciplinary intervention successfully reduced unnecessary IV antihypertensive treatment. This may serve as a model for other institutions.

Disclosures: There are no relevant conflicts of interest to disclose for any authors.

2016;176(7):981-988. doi: 10.1001/jamainternmed.2016.1509.

9. Ipek E, Oktay AA, Krim SR. Hypertensive crisis: an update on clinical approach and management. Curr Opin Cardiol. 2017;32(4):397-406. doi: 10.1097/ HCO.0000000000000398.

10. Cho HC, Dunn A, Di Capua J, Lee IT, Makhni S, Korenstein DR. Student high value care committee: a model for student-led implementation [abstract 286]. J Hosp Med. 2017

11. Yang JY, Chiu S, Krouss M. Overtreatment of asymptomatic hypertension-urgency is not an emergency: a teachable moment. JAMA Intern Med. 2018;178(5):704-705. doi: 10.1001/jamainternmed.2018.0126.

12. Malesker MA, Hilleman DE. Intravenous labetalol compared with intravenous nicardipine in the management of hypertension in critically ill patients. J Crit Care. 2012;27(5):528 e527-514. doi: 10.1016/j.jcrc.2011.12.005.

13. Hoffmann TC, Del Mar C. Clinicians' expectations of the benefits and harms of treatments, screening, and tests: a systematic review. JAMA Intern Med. 2017:177(3):407-419. doi: 10.1001/jamainternmed.2016.8254.

14. Hoffmann TC, Del Mar C. Patients' expectations of the benefits and harms of treatments, screening, and tests: a systematic review. JAMA Intern Med. 2015;175(2):274-286. doi: 10.1001/jamainternmed.2014.6016.

15. Casarett $D$. The science of choosing wisely--overcoming the therapeutic illusion. N Engl J Med. 2016;374(13):1203-1205. doi: 10.1056/NEJMp1516803.

16. Wilensky G. Changing physician behavior is harder than we thought. JAMA. 2016;316(1):21-22. doi: 10.1001/jama.2016.8019.

17. Mostofian F, Ruban C, Simunovic N, Bhandari M. Changing physician behavior: what works? Am J Manag Care. 2015;21(1):75-84.

18. Pasik $S$, Korenstein $D$, Israilov $S$, Cho HJ. Engagement in eliminating overuse: the argument for safety and beyond. J Patient Saf. 2018. doi: 10.1097/ PTS. 0000000000000487 